\title{
Correction to: Frozen blood clots can be used for the diagnosis of distinct Plasmodium species in man and non-human primates from the Brazilian Atlantic Forest
}

Filipe Vieira Santos de Abreu 1,2, Larissa Rodrigues Gomes 3,4, Aline Rosa Lavigne Mello3,4, Cesare Bianco-Júnior 3,4, Anielle de Pina-Costa ${ }^{4,5,8}$, Edmilson dos Santos ${ }^{6}$, Danilo Simonini Teixeira ${ }^{7}$, Patrícia Brasil ${ }^{4,5}$, Cláudio Tadeu Daniel-Ribeiro ${ }^{3,4}$, Ricardo Lourenço-de-Oliveira ${ }^{1,4^{*}}$ and Maria de Fátima Ferreira-da-Cruz ${ }^{3,4^{*}}$ (1)

\section{Correction to: Malar J (2018) 17:338}

https://doi.org/10.1186/s12936-018-2485-0

Following publication of the original article [1], it was flagged that one of the authors (Anielle de Pina Costa) is missing an affiliation in the article.

Please note the author Anielle de Pina Costa also has this affiliation:

Centro Universitário Serra dos Órgãos (UNIFESO), Teresópolis/RJ, 25964-004, Brazil.

The author apologizes for this error.

\section{Author details}

${ }^{1}$ Laboratório de Mosquitos Transmissores de Hematozoários, Instituto Oswaldo Cruz, Fiocruz, Rio de Janeiro, Brazil. ${ }^{2}$ Instituto Federal do Norte de Minas Gerais Campus Salinas, Minas Gerais, Brazil. ${ }^{3}$ Laboratório de Pesquisa em Malária, Instituto Oswaldo Cruz, Fiocruz, Rio de Janeiro, Brazil. ${ }^{4}$ Centro de Pesquisa, Diagnóstico e Treinamento em Malária, Instituto Oswaldo Cruz, Fiocruz, Rio de Janeiro, Brazil. ${ }^{5}$ Laboratório de Doenças Febris Agudas, Instituto Nacional de Infectologia Evandro Chagas, Fiocruz, Rio de Janeiro, Brazil. ${ }^{6}$ Divisão de Vigilância Ambiental em Saúde, Secretaria de Saúde do Rio Grande do Sul, Porto Alegre, Rio Grande do Sul, Brazil. ${ }^{7}$ Faculdade de Agronomia e Veterinárias, Universidade de Brasília, Brasília, Brazil. ${ }^{8}$ Centro Universitário Serra dos Órgãos (UNIFESO), Teresópolis, RJ 25964-004, Brazil.
The original article can be found online at https://doi.org/10.1186/s1293 6-018-2485-0

Reference

1. de Abreu FVS, Gomes LR, Mello ARL, Bianco-Júnior C, de Pina-Costa A, dos Santos E, Teixeira DS, Brasil P, Daniel-Ribeiro CT, Lourenço-deOliveira R, de Fátima Ferreira-da-Cruz M. Frozen blood clots can be used for the diagnosis of distinct Plasmodium species in man and non-human primates from the Brazilian Atlantic Fores. Malar J. 2018;17:338. https:// doi.org/10.1186/s12936-018-2485-0.

\section{Publisher's Note}

Springer Nature remains neutral with regard to jurisdictional claims in published maps and institutional affiliations.

\footnotetext{
*Correspondence: lourenco@ioc.fiocruz.br; mffcruz28@gmail.com

${ }^{4}$ Centro de Pesquisa, Diagnóstico e Treinamento em Malária, Instituto

Oswaldo Cruz, Fiocruz, Rio de Janeiro, Brazil

Full list of author information is available at the end of the article
} 